\title{
Astrophysically Motivated Laboratory Measurements of Deuterium Reacting with Isotopologues of $\mathrm{H}_{3}^{+}$
}

\author{
K.P. Bowen ${ }^{1}$, P.-M. Hillenbrand ${ }^{1}$, J. Liévin ${ }^{2}$, X. Urbain ${ }^{3}$ \\ and D.W. Savin ${ }^{1}$ \\ ${ }^{1}$ Columbia Astrophysics Laboratory, Columbia University, New York, NY, U.S.A. \\ ${ }^{2}$ Service de Chimie Quantique et Photophysique, Université Libre de Bruxelles, Brussels, \\ Belgium \\ ${ }^{3}$ Institute of Condensed Matter and Nanosciences, Université catholique de Louvain, \\ Louvain-la-Neuve, Belgium \\ email: savin@astro.columbia.edu
}

\begin{abstract}
H}_{2} \mathrm{D}^{+}$and $\mathrm{D}_{2} \mathrm{H}^{+}$are important chemical tracers of prestellar cores due to their pure rotational spectra that can be excited at the $\sim 20 \mathrm{~K}$ temperature of these environments. The use of these molecules as probes of prestellar cores requires understanding the chemistry that forms and destroys these molecules. Of the eight key reactions that have been identified (Albertsson et al. 2013), five are thought to be well understood. The remaining three are the isotope exchange reactions of atomic $\mathrm{D}$ with $\mathrm{H}_{3}^{+}, \mathrm{H}_{2} \mathrm{D}^{+}$, and $\mathrm{D}_{2} \mathrm{H}^{+}$. Semi-classical results differ from the classical Langevin calculations by an order of magnitude (Moyano et al. 2004). To resolve this discrepancy, we have carried out laboratory measurements for these three reactions. Absolute cross sections were measured using a dual-source, merged fast-beams apparatus for relative collision energies between $\sim 10 \mathrm{meV}$ to $\sim 10 \mathrm{eV}$ (Hillenbrand et al. 2019). A semi-empirical model was developed incorporating high level quantum mechanical ab initio calculations for the zero-point-energycorrected potential energy barrier in order to generate thermal rate coefficients for astrochemical models. Based on our studies, we find that these three reactions proceed too slowly at prestellar core temperatures to play a significant role in the deuteration of $\mathrm{H}_{3}^{+}$isotopologues.
\end{abstract}

Keywords. astrochemistry, ISM: molecules, methods: laboratory, molecular data, molecular processes

$\mathrm{H}_{3}^{+}$and its isotopologues play an important role in the dynamics of prestellar cores. At the densities and temperatures typical for prestellar cores, most molecules freeze onto dust grains (van der Tak 2006). A notable exception is $\mathrm{H}_{3}^{+}$and its isotopologues, which become the dominant positive charge carriers in the gas, coupling the gas to any ambient magnetic fields and thereby affecting core collapse (Kong et al. 2015). For this reason, it is highly desirable to know the total abundance of $\mathrm{H}_{3}^{+}$and its isotopologues within a prestellar core. However, the symmetric $\mathrm{H}_{3}^{+}$and $\mathrm{D}_{3}^{+}$have no pure rotational spectra that can be excited at prestellar core temperatures, and thus cannot be directly observed. This is to be contrasted with $\mathrm{H}_{2} \mathrm{D}^{+}$and $\mathrm{D}_{2} \mathrm{H}^{+}$, which have pure rotational spectra that can be excited at prestellar core temperatures, rendering them directly observable.

Using $\mathrm{H}_{2} \mathrm{D}^{+}$and $\mathrm{D}_{2} \mathrm{H}^{+}$to probe prestellar cores and infer the total abundance of the $\mathrm{H}_{3}^{+}$isotopologues requires understanding the chemistry that forms and destroys these molecules. Collisions with $\mathrm{D}, \mathrm{HD}$, and $\mathrm{D}_{2}$ are predicted to be the most important astrochemical reactions that deuterate $\mathrm{H}_{3}^{+}, \mathrm{H}_{2} \mathrm{D}^{+}$, and $\mathrm{D}_{2} \mathrm{H}^{+}$, forming $\mathrm{H}_{2} \mathrm{D}^{+}, \mathrm{D}_{2} \mathrm{H}^{+}$, and $\mathrm{D}_{3}^{+}$, respectively (Albertsson et al. 2013). Reactions with $\mathrm{HD}$ and $\mathrm{D}_{2}$ are considered relatively well understood experimentally and theoretically (Adams \& Smith 1981; Gerlich et al. 2002; Gerlich \& Schlemmer 2002; Giles et al. 1992; Hugo et al. 2009). However, there are 
no experimental measurements for deuterating reactions with atomic D. Moreover, the only published theoretical cross sections (Moyano et al. 2004) have been found to have several chemical inconsistencies (Hillenbrand et al. 2019).

To address this issue, we have carried out laboratory measurements for the three $\mathrm{H}_{3}^{+}$ isotopologues that undergo isotope exchange reactions with atomic D. We have used our dual-source, merged-beams apparatus (O'Connor et al. 2015; de Ruette et al. 2016) to measure absolute integral cross sections for relative collision energies ranging from $\sim 10$ meV to $\sim 10 \mathrm{eV}$ (Hillenbrand et al. 2019). In addition, high level quantum mechanical $a b$ initio calculations were performed to model the zero-point-energy corrected energy profiles and refine the predicted potential energy barrier. From the combined experimental and theoretical data, a semi-empirical model was developed to generate thermal rate coefficients for the reactions of $\mathrm{D}$ with the isotopologues of $\mathrm{H}_{3}^{+}$, for use in astrochemical models.

Our combined theoretical and experimental results find that there is a reaction barrier of $\sim 800 \mathrm{~K}$ for the deuterating reactions studied here. Thus, in the absence of tunneling, these reactions do not proceed at prestellar core temperatures. Taking into account the effect of tunneling results in rate coefficients of $<10^{-13} \mathrm{~cm}^{3} \mathrm{~s}^{-1}$ for temperatures below $20 \mathrm{~K}$. Hence, even with tunneling, these reactions proceed too slowly to be important at prestellar core temperatures. Based on our findings and the work of others (Hugo et al. 2009; Albertsson et al. 2013), we conclude that reactions wth HD are the primary reactions that deuterate the isotopologues of $\mathrm{H}_{3}^{+}$in prestellar cores. More details on our findings can be found in Hillenbrand et al. (2019) and Bowen et al. (in prep).

This research was supported by the NSF Division of Astronomical Sciences Astronomy Astrophysics Grants program under AST-1613267. K.P.B. was able to attend the IAU S350 thanks to a travel grant provided by the IAU and Europlanet. P.-M.H. was supported by the German Research Foundation (DFG) under grant number HI 2009/1-1. J.L. thanks the ULB/VUB computing center and the Consortium des Equipments de Calcul Intensif (FRS-FNRS and Walloon Region) for computational support. X.U. is a Senior Research Associate of the Fonds de la Recherche Scientifique-FNRS and acknowledges travel support from Fonds de la Recherche Scientifique-FNRS though IISN grant number 4.4504.10.

\section{References}

Adams, N.G., \& Smith, D. 1981, ApJ, 248, 373

Albertsson, T., Semenov, D.A., Vasyunin, A.I., Henning, Th., \& Herbst, E. 2013, ApJS, 207, 27

Bowen, K.P., Hillenbrand, P.-M., Liévin, J., Urbain, X., \& Savin, D.W., in prep.

de Ruette, N., Miller, K.A., O'Connor, A.P., Urbain, X., Buzard, C.F., Vissapragada, S., \& Savin, D.W. 2016, ApJ, 816, 31

Gerlich, D., Herbst, E., \& Rueff, E. 2002, PधSS, 50, 1275

Gerlich, D., \& Schlemmer, S. 2002, PESSS, 50, 1287

Giles, K., Adams, N.G., \& Smith, D. 1992, JPhCh, 96, 7645

Hillenbrand, P.-M., Bowen, K.P., Liévin, J., Urbain, X., \& Savin, D.W. 2019, ApJ, 877, 38

Hugo, E., Asvany, O., \& Schlemmer, S. 2009, JChPh, 130, 164302

Kong, S., Caselli, P., Tan, J.C., Wakelam, V., \& Sipilä, O. 2015, ApJ, 804, 98

Moyano, G.E., Pearson, D., \& Collins, M.A. 2004, JChPh, 121, 12396

O'Connor, A.P., Urbain, X., Stützel, J., Miller, K.A., de Ruette, N., Garrido, M., \& Savin, D.W. 2015, ApJ, 219, 6

van der Tak, F.F.S. 2006, RSPTA, 364, 3101 